\title{
Fanconi anemia pathway-deficient tumor cells are hypersensitive to inhibition of ataxia telangiectasia mutated
}

\author{
Richard D. Kennedy, Clark C. Chen, Patricia Stuckert, Elyse M. Archila, \\ Michelle A. De la Vega, Lisa A. Moreau, Akiko Shimamura, and Alan D. D'Andrea \\ Department of Radiation Oncology, Dana-Farber Cancer Institute, Harvard Medical School, Boston, Massachusetts, USA.
}

\begin{abstract}
The Fanconi anemia (FA) pathway maintains genomic stability in replicating cells. Some sporadic breast, ovarian, pancreatic, and hematological tumors are deficient in FA pathway function, resulting in sensitivity to DNA-damaging agents. FA pathway dysfunction in these tumors may result in hyperdependence on alternative DNA repair pathways that could be targeted as a treatment strategy. We used a high-throughput siRNA screening approach that identified ataxia telangiectasia mutated (ATM) as a critical kinase for FA pathway-deficient human fibroblasts. Human fibroblasts and murine embryonic fibroblasts deficient for the FA pathway were observed to have constitutive ATM activation and $\mathrm{Fancg}^{-1-} \mathrm{Atm}^{-/-}$mice were found to be nonviable. Abrogation of ATM function in FA pathway-deficient cells resulted in DNA breakage, cell cycle arrest, and apoptotic cell death. Moreover, Fanconi anemia complementation group G- (FANCG-) and FANCC-deficient pancreatic tumor lines were more sensitive to the ATM inhibitor KU-55933 than isogenic corrected lines. These data suggest that $A T M$ and $F A$ genes function in parallel and compensatory roles to maintain genomic integrity and cell viability. Pharmaceutical inhibition of ATM may have a role in the treatment of FA pathway-deficient human cancers.
\end{abstract}

\section{Introduction}

Genomic instability is an important feature in cancer progression, as it enables the activation of oncogenes, inactivation of tumor suppressor genes, and amplification of treatment-resistance genes $(1,2)$. Genomic instability is a consequence of a failure in the normal DNA damage response, which can be achieved through the loss of DNA damage signaling, checkpoint, and repair pathways (3). Disruption of DNA repair promotes an increased rate of mutagenesis, but it also renders cancer cells more susceptible to DNA damage that may occur when metabolic pathways are active or following exposure to exogenous agents such as those used in cancer therapy (5).

The Fanconi anemia (FA) pathway is one of the DNA damage response mechanisms frequently lost in cancer. FA is a rare autosomal recessive or X-linked disease characterized by developmental abnormalities, progressive bone marrow failure, and a predisposition to cancer. FA patients' cells demonstrate hypersensitivity to DNA crosslinking agents, in keeping with a role for the FA pathway in DNA repair (6). The FA pathway primarily responds to DNA damage that causes stalling of DNA replication forks during S phase. The DNA damage response kinase, ATR (ataxia telangiectasia and Rad3 related), is recruited to single-stranded DNA at stalled replication fork structures (7) and activates the FA core complex (8). The active FA core complex consists of at least 8 of the known FA proteins (A, B, C, E, F, G, L, and M) and functions as an E3 ligase that monoubiquitinates Fanconi anemia complementation group D2 (FANCD2), resulting in its association with other repair proteins, such as FANCD1/breast cancer 2,

Nonstandard abbreviations used: ATM, ataxia telangiectasia mutated; ATR, ataxia telangiectasia and Rad3 related; BER, base excision repair; DSB, double-stranded DNA break; FA, Fanconi anemia; FANC, Fanconi anemia complementation group; HR, homologous recombination; MEF, mouse embryonic fibroblast.

Conflict of interest: A.D. D'Andrea has equity exceeding \$10,000 in The DNA Repair Company Inc. (DNAR Inc.).

Citation for this article: J. Clin. Invest. 117:1440-1449 (2007). doi:10.1172/JCI31245 early onset (FANCD1/BRCA2), BRCA1, RAD51, NBS1, and PCNA, in chromatin (9-13). Monoubiquitination of FANCD2 can be easily detected by Western blotting and therefore represents a useful biomarker of FA pathway activation (5). Although the precise roles of the FA pathway in the response to DNA damage remain unclear, current data suggest that it functions to coordinate DNA repair pathways such as homologous recombination (HR) and translesion synthesis (14).

Increasing evidence suggests that loss of the FA pathway can occur in the development of cancer in patients who do not have FA. Heterozygous carriers of FA gene mutations do not suffer from FA but may have an increased risk of cancer development later in life. Heterozygous mutations in BRCA2/FANCD1 $(15,16)$, FANCJ (BRIP1) (17, 18), FANCC, FANCG $(19,20)$, and FANCA $(21,22)$ have been reported to predispose to breast, ovarian, pancreatic, and hematological malignancies. In these cancers, inactivation of the FA pathway results from loss of the remaining functional FA gene (loss of heterozygosity). In addition to mutation, epigenetic silencing of wild-type FA gene expression appears to be important in certain cancer types. Loss of expression of FANCA, FANCC (23), FANCD2 (24), FANCF (23, 25-27), and FANCG (23) have been reported in sporadic hematologic, head and neck, lung, and ovarian and cervical cancers. Taken together, these observations imply that loss of the FA pathway is associated with, and may be important for, the development of some types of sporadic cancer. Importantly, in these cases the FA pathway defect is unique to the tumor cells, while other cells, such as stem cells in the bone marrow, have an intact DNA damage repair response.

There is evidence from cell line and murine models that cancer cells deficient in DNA damage response pathways become hyperdependent on alternative pathways for survival. Inhibition of an alternative survival pathway, such as the disruption of base excision repair (BER) by poly (ADP-ribose) polymerase (PARP) inhibitors in BRCA1- or BRCA2-deficient cells, results in catastrophic 


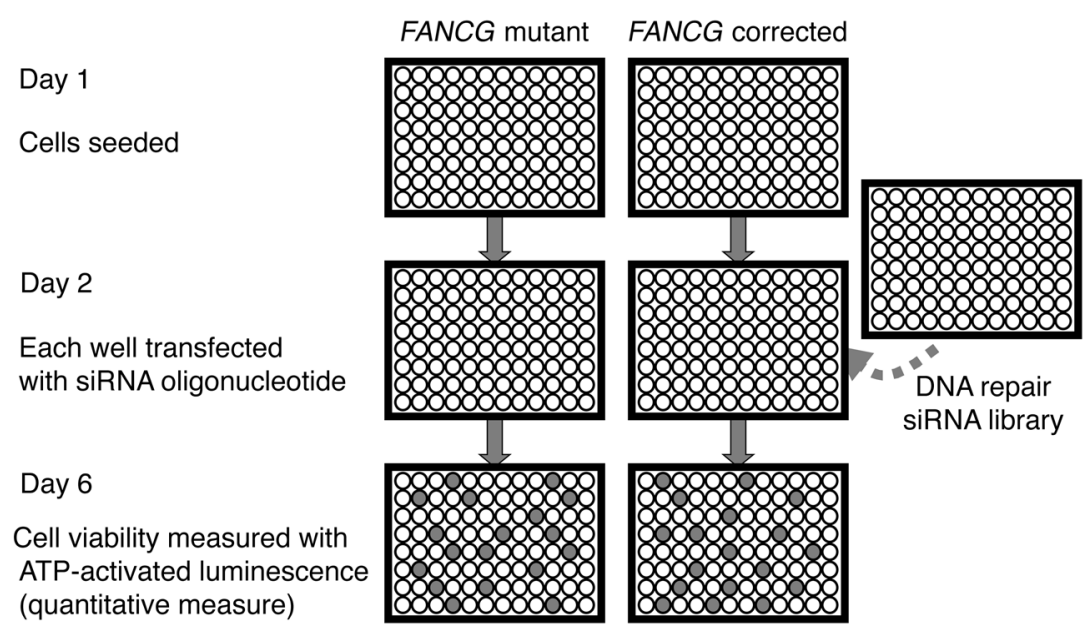

\section{Figure 1}

The identification of siRNA oligonucleotides that are selectively toxic to FA pathway-deficient cells. Cells were plated on day 1 . On day 2 , each well was transfected with an siRNA oligonucleotide directed toward 1 DNA damage response gene. On day 6, cellular viability was measured using an ATP-activated bioluminescence assay. The screen was repeated twice and the data combined for analysis.
DNA damage and cell death $(28,29)$. Since there is a clear association between FA pathway dysfunction and cancer development, we asked whether FA pathway-deficient cells are hyperdependent on other DNA damage response pathways. In order to answer this question we used a high-throughput siRNA screening approach in which we measured the relative toxicity of 460 oligonucleotides, targeted to 230 specific DNA damage response genes, in FA pathway-functional and -deficient isogenic cell lines. Using this approach we identified several gene targets that were selectively toxic to FA pathway-deficient cells. Among these targets was the DNA damage response kinase ataxia telangiectasia mutated $(A T M)$, which can be inhibited chemically as a potential cancer treatment strategy.

\section{Results}

Identification of siRNA targets that are selectively toxic to FA pathwaydeficient cells. The EUFA326 cell line is a patient-derived FANCG mutant fibroblast line that has previously been corrected with a FANCG-expressing construct to make the isogenic EUFA326G cell line (30). These paired cell lines were used for the screen because they grow at comparable rates and were found to demonstrate equal levels of gene knockdown by RT-PCR and Western blotting with siRNA oligonucleotides (data not shown).

In this screen we used the QIAGEN DNA repair siRNA library that individually targets 230 DNA damage response genes. The screening process is outlined in Figure 1 and detailed in Methods. The 10 targets that were most selectively toxic to FA pathway-deficient EUFA326 cells are given in Table 1. Knockdown of either of the BER genes, PARP1 or NEIL1, was selectively toxic to FA pathway-deficient cells, with a relative survival of $64 \% \pm 10 \%$ for PARP1 knockdown and 74\% $\pm 9 \%$ for NEIL1 knockdown compared with the corrected cell line. This is in keeping with a recent study reporting that FA pathway-deficient mouse embryonic fibroblasts (MEFs) are selectively sensitive to disruption of BER by PARP inhibitors (31) and validated our approach.

Concomitant loss of the FA pathway and ATM function is toxic to cells. Knockdown of TP53BP1 or ATM was selectively toxic to FA pathway-deficient EUFA326 cells, with a relative survival of $73 \% \pm 10 \%$ and $73 \% \pm 5 \%$ when compared with the EUFA326G cell line (Table 1 ). Knockdown of NBS1 also appeared to be selectively toxic to the EUFA326 cell line, although the result was not statistically different from that seen for the control siRNA (Table 1). NBS1 and
TP53BP1 have previously been reported to be involved in the ATMmediated response to double-stranded DNA breaks (DSBs) (32). Therefore, our data suggested a hyperdependence on the ATMmediated DSB signaling pathway in FA pathway-deficient cells.

In order to confirm that FA pathway-deficient cells require ATM function, we transfected the EUFA326 and EUFA326G cell lines with an alternative sequence $A T M$ siRNA oligonucleotide. Consistent with the screen results, the EUFA326 cell line was more sensitive to ATM knockdown, with a viability $72.8 \% \pm 3.0 \%$ (mean \pm SEM) of GFP-targeted siRNA control, compared with the EUFA326G cell line, with a viability $97.5 \% \pm 1.7 \%$ of GFPtargeted siRNA control (Figure 2A).

Since FA pathway-deficient cells were sensitive to loss of ATM expression, we asked whether the converse was true: namely, whether ATM-deficient cells are sensitive to loss of the FA pathway. ATM mutant AT22IJE-T cells and an isogenic wild-type ATM-corrected cell line (33) were transfected with siRNA targeting FANCG, and cellular viability was measured at 96 hours (Figure 2B). The ATM-deficient cell line was more sensitive to loss of FANCG expression, with a viability $58.3 \% \pm 2.2 \%$ of GFP-targeted siRNA control, compared with the corrected cell line, with a viability $73.1 \% \pm 1.6 \%$

\section{Table 1}

The 10 siRNA oligonucleotide targets that are most selectively toxic to FA pathway-deficient EUFA326 cells compared with the corrected EUFA326G cell line

$\begin{array}{lccc}\text { Rank } & \text { Gene target } & \text { Function } & \begin{array}{c}\text { EUFA326/ } \\ \text { EUFA326G } \\ \text { viability }\end{array} \\ & & & 0.59 \pm 0.09 \\ 1 & \text { TREX2 } & \text { DNA exonuclease } & 0.64 \pm 0.10 \\ 2 & A D P R T(P A R P 1) & \text { BER } & 0.68 \pm 0.18 \\ 3 & P L K 1 & \text { Cell cycle progression } & 0.70 \pm 0.14 \\ 4 & \text { UBE2B (RAD6) } & \text { Switching of DNA polymerases } & 0.70 .11 \\ 5 & \text { CDK7 } & \text { Transcription } & 0.73 \pm 0.11 \\ 6 & \text { TP53BP1 } & \text { Sensor of DSBs; activates ATM } & 0.73 \pm 0.10 \\ 7 & \text { ATM } & \text { DSB response kinase } & 0.73 \pm 0.05 \\ 8 & \text { NEIL1 } & \text { BER } & 0.74 \pm 0.09 \\ 9 & \text { RAD54B } & \text { HR } & 0.75 \pm 0.08 \\ 10 & \text { NBS1 } & \text { Sensor of DSBs; activates ATM } & 0.76 \pm 0.20 \\ \text { Control } & \text { GFP/LacZ } & \text { Control } & 0.97 \pm 0.06\end{array}$



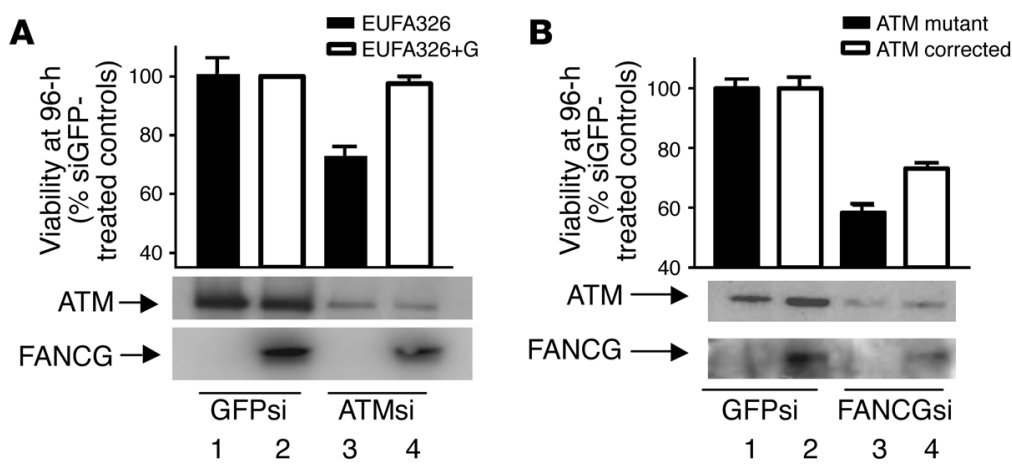

Figure 2

The combined loss of the FA pathway and ATM function is toxic to cells. (A) The EUFA326 and EUFA326G cell lines were treated for 96 hours with control GFP-targeted siRNA (lanes 1 and 2) or siRNA targeting ATM (lanes 3 and 4). Viability was measured at 96 hours, and protein was extracted and subjected Western blotting for ATM and FANCG. Viability is presented as a percentage (mean $\pm \mathrm{SEM} ; n=3$ experiments) for each well relative to the GFP siRNA treated control for each cell line. Lanes 1 and 3 represent the FA pathway-deficient EUFA326 cell line; lanes 2 and 4 of the 96-hour viability graph represent the corrected EUFA326G cell line. (B) The AT22IJE-T and AT22IJE-T-ATM cell lines were treated for 96 hours with control GFP siRNA (lanes 1 and 2) or siRNA targeting FANCG (lanes 3 and 4). Viability is presented as a percentage (mean \pm SEM; $n=3$ experiments) for each well relative to the GFP siRNA treated control for each cell line. Lanes 1 and 3 of the Western blot and bars 1 and 3 of the graph represent the ATM-deficient AT22IJE-T cell line; lanes 2 and 4 of the Western blot and bars 2 and 4 of the viability graph represent AT22IJE-T-ATM-corrected cell line.

of GFP-targeted siRNA control. A similar effect was seen with siRNA targeting FANCC in the ATM-deficient cell line (Supplemental Figure 1; supplemental material available online with this article; doi:10.1172/JCI31245DS1). These data further support the hypothesis that the concomitant loss of both the FA pathway and ATM function is toxic to cells.

Double knockout of murine Fancg and Atm results in embryonic lethality. Next we investigated the interaction between the FA and ATM pathways through the interbreeding of $\mathrm{Fancg}^{+/-} \mathrm{Atm}^{+/-}$mice. A consistent observation was the absence of $\mathrm{Fancg}^{-1-} \mathrm{Atm}^{-1-}$ offspring (Table 2). The absence of a $\mathrm{Fancg}^{-1-} \mathrm{Atm}^{-/-}$genotype was highly statistically significant $(P<0.001)$ when compared with the frequency of $6.25 \%$ predicted by Mendelian distribution. These data suggest that cells do not tolerate a loss of ATM and FA pathway function in utero and are consistent with siRNA screen results. $\mathrm{Fancg}^{+/-} \mathrm{Atm}^{-/-}$and $\mathrm{Fancg}^{-/-} \mathrm{Atm}^{+/-}$ progeny were also slightly less frequent than would have been expected by Mendelian genetics (8.54\% compared with $12.50 \%$ and $10.68 \%$ compared with $12.50 \%$, respectively), although these differences were not statistically significant (Table 2).

FA pathway-deficient cells require constitutive activation of ATM to prevent accumulation of spontaneous DNA breakage. The siRNA library and murine data suggested a specific requirement for ATM in the absence of the FA pathway. To study this further, we investigated ATM function in FA pathway-deficient cells. Autophosphorylation of Ser1981 on ATM has previously been reported as a marker of ATM activation (34). Therefore, we compared isogenic pairs of FA pathway-functional and -deficient cells for ATM autophosphorylation by Western blotting. The FA-deficient cell lines EUFA326 (FANCG mutant), EUFA426 (FANCC mutant), and GM6914 (FANCA mutant) demonstrated constitutive activation of ATM (Figure 3A, lanes 1, 3, and 5) when compared with the isogenic corrected cell lines EUFA326+ FANCG, EUFA426 + FANCC, and GM6914 + FANCA (Figure 3A, lanes 2, 4, and 6). Phosphorylation of histone $\mathrm{H} 2 \mathrm{AX}$, a marker of DSBs, was also elevated in the FA pathway-deficient cells. A baseline level of FANCD2 monoubiquitination confirmed normal FA pathway function in each corrected cell line when compared with the corresponding FA pathway-deficient cell line (Figure 3A, compare lanes 2, 4, and 6 with lanes 1,3 , and 5). We also examined Atm activation and $\mathrm{H} 2 \mathrm{ax}$ phosphorylation in primary MEFS derived from $\mathrm{Fancg}^{-1}$ mice versus MEFs taken from Fancg wild-type mice (Figure 3B). Consistent with results from the human fibroblast lines, Fancg-deficient MEFS exhibited a basal activation of Atm and phosphorylation of H2ax (Figure 3B, lane 1) when compared with Fancg wild-type MEFs (Figure 3B, lane 2). Basal monoubiquitination of Fancd2 was also observed in the $\mathrm{Fancg}^{+/+}$MEFs when compared with the $\mathrm{Fancg}^{-/}$cell line, indicating a functional FA pathway in the wildtype cells (Figure 3B, compare lane 2 with lane 1).

To further confirm constitutive activation of ATM in FA cells, we performed in vitro kinase assays for ATM activity in EUFA326 and EUFA326G cell lines. In keeping with the Western blot data, ATM immunoprecipitated from FANCG mutant

Table 2

Frequency of genotypes of offspring from interbred $\mathrm{Fancg}^{+-} \mathrm{Atm}^{+-}$mice compared with the predicted frequencies by Mendelian genetics

\begin{tabular}{|c|c|c|c|c|c|}
\hline $\begin{array}{l}\text { Atm } \\
\text { status }\end{array}$ & $\begin{array}{l}\text { Fancg } \\
\text { status }\end{array}$ & $\begin{array}{l}\text { No. of offspring } \\
\text { observed }\end{array}$ & $\%$ Litter & $\begin{array}{c}\% \text { Predicted by } \\
\text { Mendelian distribution }\end{array}$ & $\begin{array}{l}\text { Significant difference between } \\
\text { observed and predicted? }\end{array}$ \\
\hline$+/+$ & $+/+$ & 34 & 12.10 & 6.25 & Yes $(P=0.028)$ \\
\hline$+/+$ & $+1-$ & 40 & 14.23 & 12.50 & No $(P=0.620)$ \\
\hline +/+ & $-1-$ & 16 & 5.69 & 6.25 & No $(P=0.860)$ \\
\hline+- & $+/+$ & 46 & 16.37 & 12.50 & No $(P=0.230)$ \\
\hline$+/-$ & $+/-$ & 72 & 25.62 & 25.00 & No $(P=0.923)$ \\
\hline$+/-$ & $-1-$ & 24 & 8.54 & 12.50 & No $(P=0.168)$ \\
\hline$-1-$ & $+/+$ & 19 & 6.76 & 6.25 & No $(P=0.865)$ \\
\hline$-1-$ & $+/-$ & 30 & 10.68 & 12.50 & No $(P=0.598)$ \\
\hline$-1-$ & $-1-$ & 0 & 0.00 & 6.25 & Yes $(P<0.001)$ \\
\hline
\end{tabular}




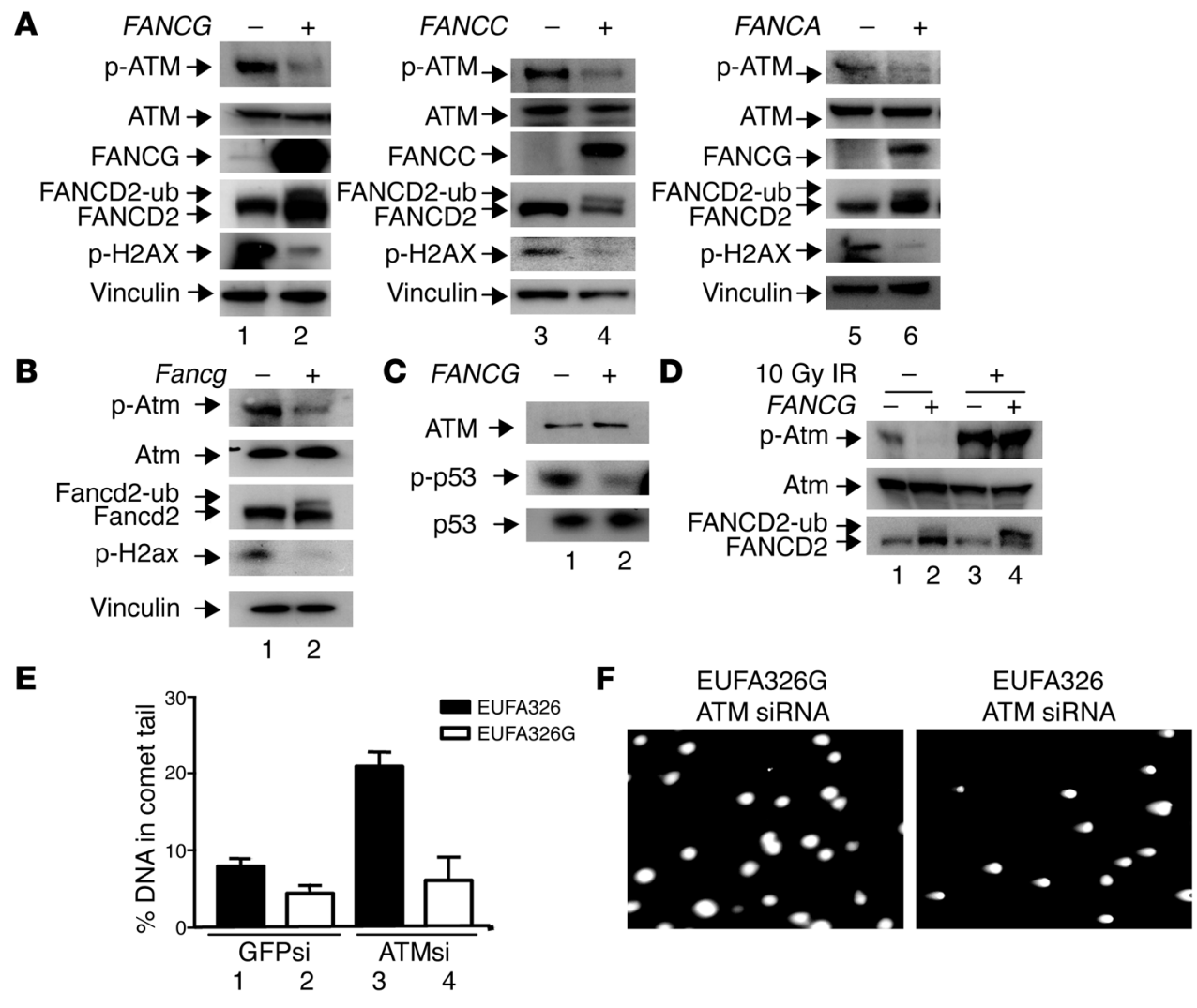

Figure 3

FA pathway-deficient cells demonstrate constitutive activation of ATM. (A) Western blots comparing activation of ATM (autophosphorylation at Ser1981), histone H2AX phosphorylation at Ser139, and monoubiquitination of FANCD2 (FANCD2-ub; slower-migrating form) in FA pathway-deficient EUFA326 (FANCG mutant), EUFA426 (FANCC mutant), GM6914 (FANCA mutant) (lanes 1, 3, and 5) and isogenic corrected (lanes 2, 4, and 6) fibroblasts. (B) A Western blot comparing activation of Atm (Ser1987 phosphorylation), histone $\mathrm{H} 2 \mathrm{ax}$ phosphorylation, and monoubiquitination of Fancd2 in the Fancg $\mathrm{F}^{-/} \mathrm{MEF}$ line (lane 1) and the Fancg ${ }^{+/+}$MEF line (lane 2). (C) A kinase assay comparing the activity of ATM extracted from FA pathway-deficient EUFA326 and corrected EUFA326G cells toward Ser15 on recombinant p53. (D) Western blot comparing ATM phosphorylation and FANCD2 monoubiquitination in FA pathway-deficient EUFA326 cells (lanes 1 and 3) and corrected EUFA326G cells (lanes 2 and 4 ) at baseline (lanes 1 and 2) and 3 hours after 10 Gy ionizing radiation (IR; lanes 3 and 4). (E) Neutral comet assay. The $y$ axis represents mean percentage of DNA in the comet tail. Black bars represent the FANCG mutant EUFA326 cell line. White bars represent the corrected EUFA326G cell line. For bars 1 and 2, cells were treated with control GFP-targeted siRNA. For bars 3 and 4, cells were treated with siRNA targeting ATM. (F) Representative comet assay fields of the EUFA326G and EUFA326 cell lines treated with siRNA targeted to ATM. DNA is stained with DAPI.

EUFA326 cells induced phosphorylation of recombinant p53 (Figure 3C, lane 1) when compared with ATM extracted from corrected EUFA326G cells (Figure 3C, lane 2).

Next we asked whether increased ATM activation in FA pathwaydeficient cells was due to dysregulation of ATM function. ATM is strongly activated by DSBs induced by ionizing radiation; therefore, we irradiated the EUFA326 and EUFA326G cells with 10 Gy of ionizing radiation and measured ATM autophosphorylation (Figure 3D). Both cell lines demonstrated normal DNA damage-induced ATM activation (Figure 3D, compare lanes 1 and 2 with lanes 3 and 4).

ATM is important for the correct response to DSBs. Therefore, we asked whether constitutive ATM activation in FA pathway-deficient cells is required for the repair of spontaneous DNA breaks. To address this question, we treated the FANCG-deficient EUFA326 and the corrected EUFA326G cell lines with siRNA targeting ATM or a control GFP sequence and assessed DNA breakage using a neutral comet assay. In this assay, the percentage of cellular DNA in the comet tail is a measure of the number of DNA breaks (35). Following transfection with control siRNA, the EUFA326 cell line demonstrated a 1.8-fold greater number of DNA breaks compared with the corrected EUFA326G cell line (Figure 3E, compare bars 1 and 2). This is consistent with the spontaneous DNA breakage characteristic of FA pathway-deficient cells (6) and the observed baseline phosphorylation of $\mathrm{H} 2 \mathrm{AX}$ reported in Figure 3A. Following treatment with siRNA targeting $A T M$, the EUFA326 cell line demonstrated a 2.6-fold increase in DNA breaks when compared to the same cell line treated with control siRNA. In contrast, the EUFA326G cell line demonstrated only a 1.4-fold increase in DNA breaks when compared to control siRNA treatment (Figure 3E, compare bars 3 and 4). Figure 3F shows representative microscopy fields demonstrating comet tails in the EUFA326 cell line compared with the EUFA326G cell line following knockdown of ATM. These data suggest that constitutive ATM activation in FA pathway-deficient cells is necessary to prevent the accumulation of sporadic DNA breaks.

FA pathway-deficient cells are sensitive to the ATM inbibitor $K U$-55933. The compound KU-55933 has recently been reported to be a highly specific, competitive ATP-binding site inhibitor of ATM (36). Since FA pathway-deficient cells were dependent on ATM function, we reasoned that they should be selectively sensitive to treatment with KU-55933. To test this hypothesis, we compared the dose viability curves of isogenic pairs of FA pathway-deficient and corrected cell lines treated with increasing doses of KU-55933. Specifically, we compared the response to KU-55933 in the FANCC mutant and corrected EUFA426 cell line, the FANCG mutant and corrected EUFA326 cell line, the FANCD2 mutant and corrected PD20 cell line, and the FANCE mutant and corrected DF1179 cell line (Figure 4A). In addition, we tested the sensitivity of $\mathrm{Fancg}^{-1} \mathrm{MEFs}$ to KU-55933 when compared with wild-type MEFs (Figure 4A). In each case the FA pathway-deficient cell line was more sensitive to KU-55933. The effective dose range for KU-55933 in each case was between $5 \mu \mathrm{M}$ and $20 \mu \mathrm{M}$, which corresponds to the previously pub- 
A
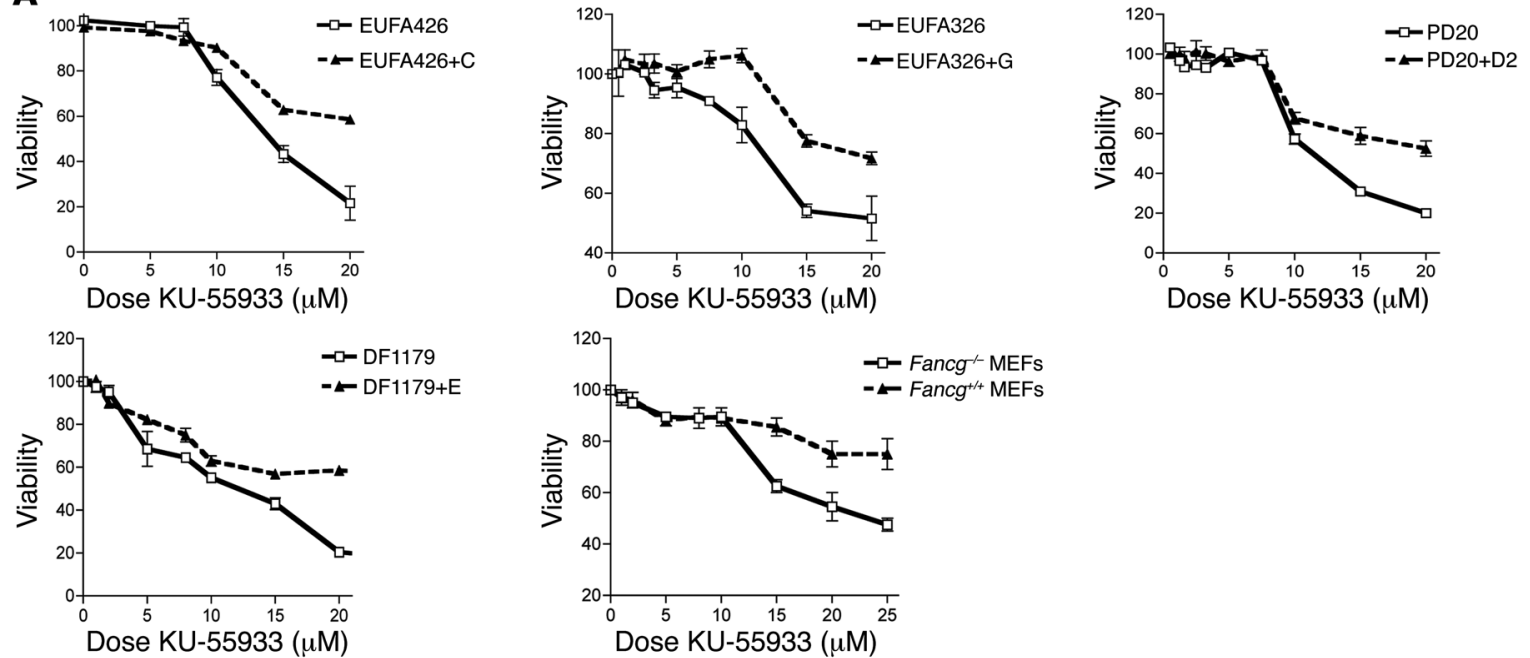

B

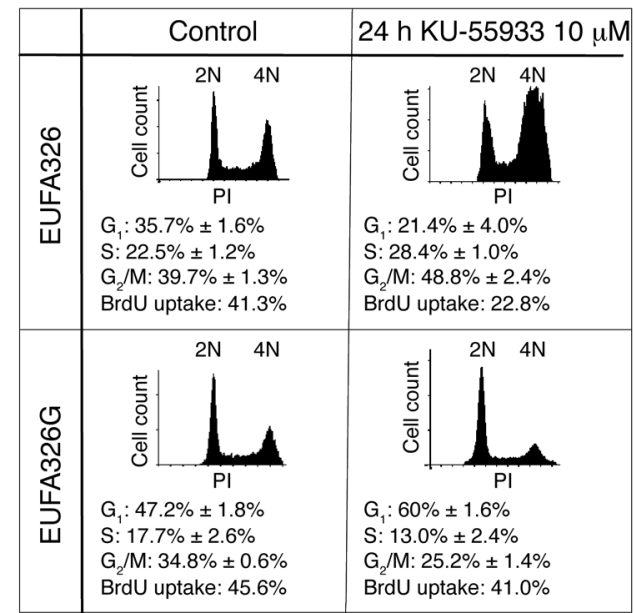

D

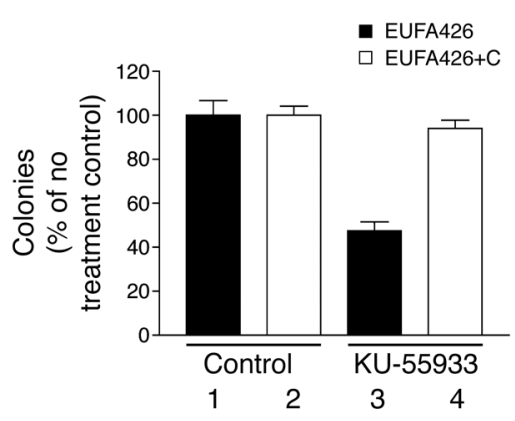

C

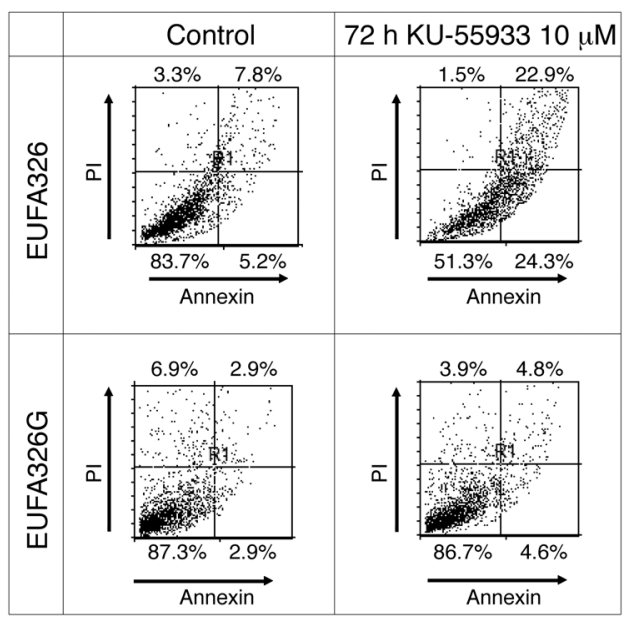

E
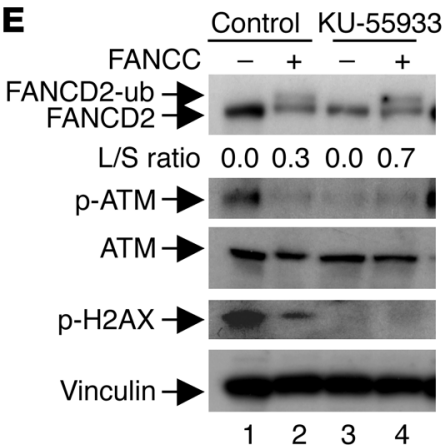

Figure 4

FA pathway-deficient cells are selectively sensitive to the ATM inhibitor KU-55933. (A) Seventy-two-hour dose viability curves comparing the response to KU-55933 for FANCC mutant EUFA426 cells, FANCG mutant EUFA326 cells, FANCD2 mutant PD20 cells, FANCE mutant DF1179 cells, and Fanc ${ }^{-1-}$ MEFs (solid lines) versus isogenic corrected cell lines (dotted lines). (B) Cell cycle profile and 1-hour BRDU uptake in FANCG mutant EUFA326 cells and corrected EUFA326G cells treated with $10 \mu \mathrm{M}$ KU-55933 for 24 hours or DMSO alone. The $x$ axis represents DNA content (PI staining). Percentages represent proportion of cells in the $\mathrm{G}_{1}(2 \mathrm{~N})$, $S$ (between $2 \mathrm{~N}$ and $4 \mathrm{~N}$ ), and $\mathrm{G}_{2} / \mathrm{M}(4 \mathrm{~N})$ phases of the cell cycle.

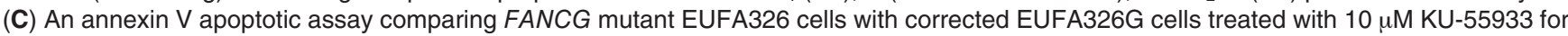
72 hours or DMSO alone. The $x$ axis represents annexin V detection. (D) A clonogenic assay comparing FANCC mutant EUFA426 cells with isogenic corrected cells 14 days after KU-55933 treatment. Lanes 1 and 2 represent untreated controls. Colony counts are given as a percentage of the untreated control for each cell line. (E) Western blot comparing FANCD2 monoubiquitination, ATM autophosphorylation, and H2AX phosphorylation in FANCC mutant EUFA426 cell line (lanes 1 and 3) and isogenic FANCC-corrected cell line (lanes 2 and 4 ). Lanes 1 and 2 represent an untreated control. Lanes 3 and 4 represent cells treated for 24 hours with $10 \mu \mathrm{M} \mathrm{KU}-55933$. L/S ratio, ratio between FANCD2-ub band (L) and FANCD2 band (S) as measured by densitometry. 


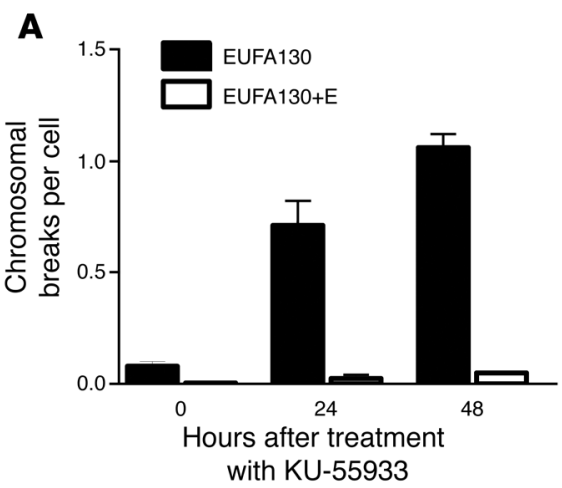

B

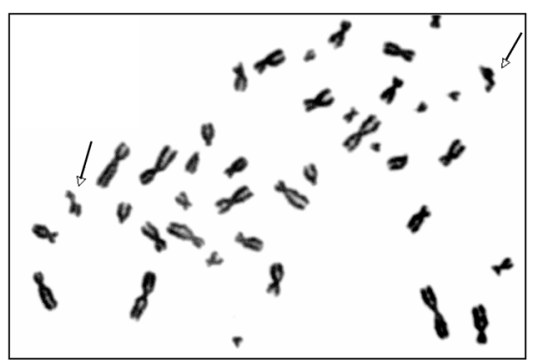

C

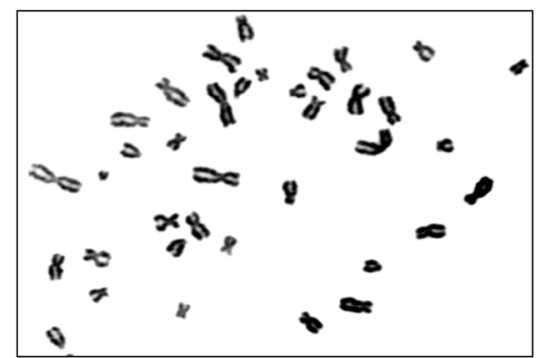

Figure 5

$\mathrm{KU}-55933$ treatment results in selective chromosomal breakage and cell death in FA pathway-deficient cells. (A) Graphical representation of the number of chromosomal breaks per cell as measured on metaphase spreads 0,24 , and 48 hours after treatment with $10 \mu \mathrm{M} \mathrm{KU}-55933$. The black bars represent the FANCE mutant EUFA130 cell line. The white bars represent the isogenic FANCE-corrected cell line. Mean values were calculated from 3 separate experiments, and the SEM is represented for each bar. (B) Photograph of representative metaphase spread 48 hours following KU-55933 treatment in for FANCE mutant EUFA130 cells. Chromosomal breaks are indicated by arrows. (C) Photograph of representative metaphase spread 48 hours following KU-55933 treatment for FANCE-corrected EUFA130E cells. Original magnification, $\times 100$.

lished concentration range for specific ATM inhibition (36). For the majority of the fibroblast cell lines tested, the greatest difference in sensitivity between FA pathway-deficient and functional cells was seen at a concentration of $20 \mu \mathrm{M} \mathrm{KU}-55933$, which also resulted in the highest level of ATM inhibition (Supplemental Figure 2A).

Decreased cellular viability may be due to cell death, cellular senescence, or impaired growth. Therefore, we investigated the effects of ATM inhibition on cell cycle profile, DNA synthesis, and cell death in FA pathway-deficient cells. EUFA326 and EUFA326G cells were treated for 24 hours with $10 \mu \mathrm{M}$ KU-55933, after which DNA synthesis and cell cycle profile were measured by flow cytometry using propidium iodide staining and BrdU uptake (Figure 4B). The FANCG mutant EUFA326 cell line accumulated in the late $\mathrm{S}$ and $\mathrm{G}_{2} / \mathrm{M}(4 \mathrm{~N})$ phase of the cell cycle (Figure $4 \mathrm{~B}$, compare panels the top 2 panels) with a $45 \%$ reduction in DNA synthesis, consistent with a failure of DNA replication during $S$ phase. In the case of the corrected EUFA326G cell line, KU-55933 treatment had a smaller effect on DNA synthesis in (a 10\% reduction), and a relative accumulation in $\mathrm{G}_{1}(2 \mathrm{~N})$ was observed. A similar $4 \mathrm{~N}$ cell cycle arrest after KU-55933 treatment was seen in the FANCA, FANCC, and FANCE mutant cell lines (data not shown).

Annexin $\mathrm{V}$ is expressed on the cell surface during apoptosis and can be detected by flow cytometry using an FITC-antibody conjugate (37). After 72 hours treatment with KU-55933, the EUFA326 cell line demonstrated increased surface staining of annexin $\mathrm{V}$ alone (increased from $5.2 \%$ to $24.3 \%$ ), indicating early apoptosis, as well as increased staining for both annexin $\mathrm{V}$ and propidium iodide (increased from $1.5 \%$ to $22.9 \%$ ), consistent with the later stages of apoptosis when the plasma membrane is permeable (Figure 4C, top panels). The FA pathway-proficient EUFA326G cell line did not demonstrate evidence of apoptotic cell death following KU-55933 treatment (Figure 4C, bottom panels).
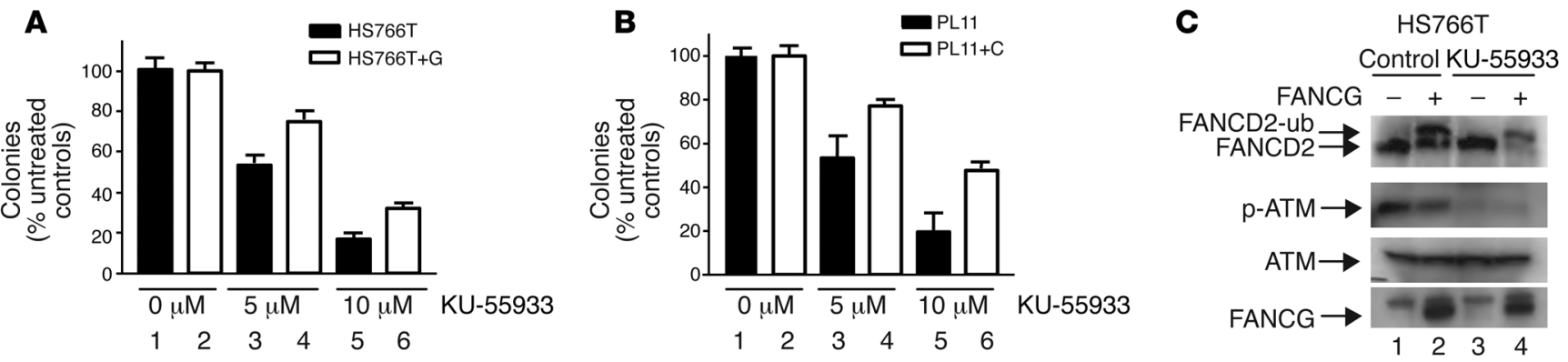

Figure 6

The FA pathway-deficient HS766T pancreatic cancer cell line is sensitive to KU-55933. (A) A clonogenic assay comparing the number of colonies in FANCG mutant HS766T cells (black bars) and the isogenic FANCG-corrected cell line (white bars) 14 days after KU-55933 treatment. Bars 1 and 2 represent untreated controls. Bars 3 and 4 represent cells treated with $5 \mu \mathrm{M} \mathrm{KU}-55933$. Bars 5 and 6 represent cells treated with $10 \mu \mathrm{M} \mathrm{KU}-55933$. Colony counts are shown as a percentage of the untreated control for each cell line. (B) A clonogenic assay comparing the number of colonies in FANCC mutant PL11 cells (black bars) and the isogenic FANCC-corrected cell line (white bars) 14 days after KU-55933 treatment. Bars 1 and 2 represent untreated controls. Bars 3 and 4 represent cells treated with $5 \mu \mathrm{M}$ KU-55933. Bars 5 and 6 represent cells treated with $10 \mu \mathrm{M} \mathrm{KU}-55933$. Colony counts are shown as a percentage of the untreated control for each cell line. (C) A Western blot comparing FANCD2 monoubiquitination and ATM autophosphorylation in the FANCG mutant HS766T cell line (lanes 1 and 3) and the isogenic FANCGcorrected cell line (lanes 2 and 4). Lanes 1 and 2 represent an untreated control. Lanes 3 and 4 represent cells treated for 24 hours with $10 \mu M$ $\mathrm{KU}-55933$. The upper band in the FANCG blot is a nonspecific cross-reacting band. 
To ensure that the specific sensitivity of FA pathway-deficient cells to KU-55933 was not assay and time dependent, clonogenic assays were performed using FANCC mutant and corrected EUFA426 cells. These cell lines were chosen because they form distinct colonies when seeded at low density. Each cell line was treated with $10 \mu \mathrm{M}$ KU-55933 for 24 hours and then seeded for colony count assays. A proportion of the KU-55933-treated EUFA426 cells was exposed to $10 \mathrm{~Gy}$ of ionizing radiation and failed to phosphorylate ATM and the ATM substrate CHK2, indicating that the inhibitor was still active at 24 hours (Supplemental Figure 2B). After 14 days, the FANCC mutant cell line was more sensitive to KU-55933, with a colony count $47.4 \% \pm 4.2 \%$ of the nontreated control, compared with the treated FA pathway-proficient EUFA426 plus FANCC (EUFA426+C) cell line, which had a colony count $94.0 \% \pm 3.7 \%$ of the nontreated control (Figure 4D).

At the same time as EUFA426 and EUFA426C cells were seeded for the colony count assay, a proportion of these cells were collected for analysis of ATM and FANCD2 activity by Western blotting. Following treatment with KU-55933, ATM phosphorylation was no longer observed in the EUFA426 cell line, indicating complete inhibition (Figure 4E, compare lanes 1 and 3). Interestingly, $\mathrm{H} 2 \mathrm{AX}$ phosphorylation was also decreased, suggesting that ATM is primarily responsible for $\mathrm{H} 2 \mathrm{AX}$ phosphorylation in this $\mathrm{FA}$ pathway-deficient cell line (Figure 4E, compare lanes 1 and 3). We also observed an increase in FANCD2 monoubiquitination in the FA pathway-functional EUFA426C cell line following KU55933 treatment (Figure 4E, lane 4) when compared with the untreated control (Figure 4E, lane 2). This observation supports the hypothesis that the FA pathway is activated following inhibition of ATM activity by KU-55933.

KU-55933 treatment results in chromosomal breakage in FA pathwaydeficient cells. A consistent feature of FA pathway-deficient cells is sporadic and DNA damage-induced chromosomal breakage (6). Since ATM primarily responds to DSBs and our data suggested a requirement for this kinase in FA pathway-deficient cells, we hypothesized that these cells may demonstrate increased chromosomal breakage following treatment with KU-55933. The FANCE-corrected cell line EUFA130+E demonstrates a low amount of mitomycin $\mathrm{C}$-induced (MMC-induced) chromosomal breakage and radial formation when compared with the FANCE mutant EUFA130 cell line, indicating an effective correction of the FA pathway (Supplemental Figure 3, A and B). Therefore, these paired cell lines were ideal for measuring the effect of FA pathway status on genomic stability following KU-55933 treatment. Each cell line was treated with $20 \mu \mathrm{M}$ KU-55933 for 24 and 48 hours, and chromosomal breakage was assessed by microscopy on metaphase spreads (Figure 5). Prior to treatment, we observed a modest but significantly greater number of chromosomal breaks per cell in the EUFA130 cell line when compared with the corrected EUFA130+E cell line $(0.080 \pm 0.02$ compared with $0.005 \pm 0.005$ ), consistent with the spontaneous chromosomal damage characteristic of FA pathway-deficient cells. After 24 hours and 48 hours of KU-55933 treatment, the EUFA130 cell line demonstrated a 9 -fold $(0.710 \pm 0.110$ breaks $)$ and 12 -fold $(1.060 \pm 0.060$ breaks $)$ increase in chromosomal breaks per cell, respectively, whereas the EUFA130E cell line showed no significant change in chromosomal breakage from baseline. When FANCG mutant EUFA326 cells were treated with siRNA targeting ATM, there was a 4-fold increase in chromosomal breakage when compared with that of the corrected EUFA326G cell line, indicating that ATM-targeted siRNA and KU-55933 have similar genotoxic effects on FA pathway-deficient cells (Supplemental Figure 4). Another feature of FA pathway-deficient cells is the formation of radial chromosomal aberrations following treatment with DNA-damaging agents. Interestingly, in contrast to treatment with DNA-damaging agents such as MMC (Supplemental Figure 3B), there was no increase in radial formation in the EUFA130 cell line following KU-55933 treatment, most likely reflecting this agent's unique mode of action.

FA pathway-deficient pancreatic cancer cell lines are sensitive to $K U$-55933. Since FA pathway-deficient cells were sensitive to KU-55933, we asked whether FA pathway-deficient cancer cell lines would also be sensitive to ATM inhibition. The HS766T and PL11 cell lines are derived from human pancreatic tumors and have mutation of 1 allele and loss of heterozygosity of the other allele of FANCG and FANCC, respectively. We treated each pancreatic cancer cell line and an isogenic cell line expressing the respective wild-type FA gene with KU-55933 and compared survival using clonogenic survival assays. In keeping with the cell lines derived from FA patients, the FA pathway-deficient HS766T cell line was more sensitive to $10 \mu \mathrm{M} \mathrm{KU}-55933$, with a colony count $16.9 \% \pm 3.2 \%$ of the untreated control compared with $32.1 \% \pm 2.0 \%$ for the corrected HS766T $+\mathrm{G}$ cell line (Figure 6A, bars 5 and 6). Similarly, the FANCC mutant PL11 cell line was more sensitive to $10 \mu \mathrm{M}$ KU-55933 with a colony count $19.6 \% \pm 8.9 \%$ of the untreated control compared with $47.5 \% \pm 3.9 \%$ for the FANCC-corrected PL11+C cell line (Figure 6B, bars 5 and 6). Increased sensitivity in both the FA pathway-deficient tumor cell lines was also seen at the lower dose of $5 \mu \mathrm{M} \mathrm{KU}-55933$. The HS766T cell line had a colony count $53.3 \% \pm 5.0 \%$ of untreated control compared with $74.9 \% \pm 5.5 \%$ for the HS766T $+\mathrm{G}$ cell line (Figure $6 \mathrm{~A}$, bars 3 and 4 ) and the PL- 11 cell line had a colony count $53.2 \% \pm 10.5 \%$ of untreated control compared with $77.0 \% \pm 2.5 \%$ for the PL $11+\mathrm{C}$ cell line (Figure 6B, bars 3 and 4). Lysates were extracted from the HS766T and HS766T+G cell lines 24 hours after treatment with $10 \mu \mathrm{M}$ KU-55933 and analyzed by Western blotting (Figure 6C). Similar to cells derived from FA patients, the FANCG mutant HS766T cell line demonstrated baseline ATM autophosphorylation (Figure 6C, lane 1). Constitutive ATM activation was reduced by FANCG correction but not completely suppressed (Figure 6C, lane 2). As expected, treatment with KU-55933 completely inactivated ATM in each cell line (Figure 6C, lanes 3 and 4). In the FANCG-corrected HS766T $+\mathrm{G}$ cell line, KU-55933 treatment resulted in increased FANCD2 monoubiquitination, suggesting a compensatory activation of the FA pathway (Figure 6C, lane 4).

\section{Discussion}

Although cytotoxic chemotherapy has proven effective in curing or palliating cancer, it damages normal replicating cells, resulting in significant side effects. Therefore, there is much interest in the development of treatments that specifically target cancer cells but have little or no effect on normal cells (38). One approach is to target DNA damage response pathways. This strategy relies on the frequency of DNA repair defects observed in cancer. Indeed, loss of DNA repair may be a prerequisite of cancer development as it results in genomic instability (2). Alternative DNA damage response pathways may be required to allow malignant cells to survive in the presence of endogenous genotoxic stress. Therefore, inhibitors of these pathways may offer an attractive strategy for the prevention or treatment of cancer (39). 
The validity of targeting DNA response pathways in cancer treatment has recently been demonstrated by Bryant et al. (29) and Farmer et al. (28). In these seminal preclinical studies, inhibition of PARP1, a component of BER, resulted in specific toxicity to BRCA1- and BRCA2-deficient (and therefore HR-defective) cells while having little effect on cells competent for DNA repair. Loss of the FA pathway has been reported in a number of different tumor types; therefore, we asked whether inhibition of alternative DNA damage response pathways could be selectively toxic to cells that have lost FA pathway function. Using an siRNA screening approach, we identified the DNA damage response kinase, ATM, as being required for the survival of FA pathway-deficient cells (Table 1 and Figure 2A). Moreover, ATM-deficient cells were sensitive to loss of FANCG expression (Figure 2B), suggesting that the combined loss of both ATM and FA pathway function is toxic to cells. Interestingly, abrogation of the FA pathway also demonstrated some toxicity to the ATM-corrected cell line. This may be explained by a failure to completely correct the AT22IJE-T cell line with exogenous ATM expression, although normal ATM-mediated phosphorylation of SMC1 was noted after ionizing radiation damage in the corrected cell line (Supplemental Figure 1B). Alternatively, loss of the FA pathway may adversely affect cell growth in the corrected cell line by introducing genomic instability that requires ATM-mediated checkpoint activation and repair.

FA pathway-deficient cells were found to have constitutive activation of ATM (Figure 3, A and B), although the level of activation was relatively low when compared with that of irradiated cells, indicating that these cells have a low level of sporadic DNA damage (Figure 3D). H2AX phosphorylation $(\gamma-\mathrm{H} 2 \mathrm{AX})$, a marker of DSBs, was also observed in FA pathway-deficient cells (Figure 3, A and B). Recent studies have demonstrated that Xenopus egg extracts immunodepleted for FANCA or FANCD2 have a higher level of spontaneous DSBs during DNA replication when compared with normal cells (40). Therefore, it seems likely that the constitutive ATM activation and H2AX phosphorylation observed in FA pathway-deficient cell lines is in response to spontaneous S-phase DNA breaks. The observation that siRNA-mediated inhibition of ATM resulted in a selective loss of viability in FA pathway-deficient cells (Figure 2A) suggests that constitutive activation of ATM, although relatively low, is critical for cell survival. Inhibition of ATM in FA pathway-deficient cells resulted in decreased DNA synthesis and a late $S / G_{2}$ cell cycle accumulation (Figure $4 B$ ), further supporting the hypothesis that FA pathway-deficient cells require ATM to reestablish normal DNA synthesis following spontaneous DNA damage. Indeed, a similar late $S / G_{2}$ accumulation is seen when cells derived from FA patients are treated with DNA-damaging agents such as melphalan, a phenotype that has been attributed to a failure to restart stalled DNA replication $(41,42)$. The nonviability of $\mathrm{Fancg}^{-1-} \mathrm{Atm}^{-/-}$mice (Table 2) further emphasizes the importance of ATM function in the context of a deficient FA pathway and, conversely, the requirement for the FA pathway in absence of ATM function. Importantly, the embryonic lethality observed in the murine model system also demonstrates that the lethal phenotype associated with ATM and FA pathway loss is not specific to tissue culture models.

Until recently, pharmacological inhibition of ATM was relatively nonspecific with drugs such as wortmannin that also target ATR and DNA-PK (43). The specific ATM inhibitor KU-55933 has been recently developed and shown not to target other PI3Krelated kinases at effective concentrations, making it a potentially useful therapeutic compound (36). Therefore, we asked whether this drug was selectively toxic to FA pathway-defective cells. Consistent with the siRNA data, FA pathway-deficient fibroblasts were sensitive to ATM inhibition by KU-55933, as measured by dose response, cell cycle, DNA synthesis, and apoptotic assays (Figure 4). Moreover, the FANCG mutant HS766T and FANCC mutant PL11 pancreatic cell lines were more sensitive to KU-55933 than the respective isogenic corrected cell lines (Figure 6, $A$ and B). However, the HS766T $+\mathrm{G}$ cell line continued to exhibit constitutive ATM activation following correction with wild-type FANCG, indicating that sporadic DNA damage was still occurring (Figure 6C, lanes 1 and 2). The PL11+C cell line demonstrated a similar level of ATM activation after correction with FANCC (data not shown). This is most likely due to the loss of other DNA damage response pathways in the development of these cancer cells and may account for the relative sensitivity of the corrected $\mathrm{HS766T}+\mathrm{G}$ and PL11+C cell lines to KU-55933 when compared with the FA pathway-corrected fibroblast cell lines.

KU-55933 treatment of FA pathway-deficient EUFA130 cells compared with the corrected cell line resulted in marked chromosomal breakage (Figure 5). These chromosomal breaks represent the persistence of DSBs that have not been repaired during S phase. Importantly, KU-55933 treatment did not induce significant chromosomal breakage in the FA pathway-proficient EUFA130+E cell line (Figure 5), suggesting that either the FA pathway can reestablish replication forks efficiently without double-stranded DNA breakage or the FA pathway is able to repair DSBs independent of ATM. Interestingly, FA pathway-deficient cells underwent apoptosis following KU-55933 treatment, indicating activation of $A T M$-independent programmed cell death in response to persistent DNA breaks (Figure 4C).

Our group has previously shown that ionizing radiation results in ATM-mediated phosphorylation of FANCD2 and S-phase checkpoint activation independent of the FA core complex and monoubiquitination of FANCD2 (44). Our current data support a model wherein the FA core complex responds to sporadic S-phase DNA damage through the monoubiquitination of FANCD2 in a pathway parallel to ATM function. In normal cells, sporadic DNA breaks cause DNA replication fork stalling that activates ATR and then the FA pathway. The FA pathway, in turn, stabilizes and coordinates repair at the fork. An alternative pathway also exists in which the stalled fork collapses to form a DSB that activates ATM. ATM then phosphorylates mediator proteins that result in cell cycle arrest and repair. In FA pathway-deficient cells, stalled forks are primarily repaired through an ATM-dependent pathway. Although the exact role for ATM in the response to a stalled DNA replication fork is unclear, it may activate DNA damage response checkpoints that allow time for efficient repair through HR or nonhomologous end-joining (NHEJ). Recent data also suggest that ATM may have a direct signaling role to NHEJ (45) or a role in HR (46).

The ATM-mediated pathway may be less efficient in the absence of the FA pathway, accounting for the sporadic chromosomal breaks seen in FA cells, but is sufficient to allow the majority of cells to survive. However, the addition of ATM-targeted siRNA or KU-55933 to FA pathway-deficient cells inhibits the ATM-mediated pathway, leaving no alternative mechanism of repair. This results in persistent DSBs, failed DNA synthesis, chromosomal damage, and ultimately cell death. 
In conclusion, we present data demonstrating a specific requirement for $A T M$ function in cells deficient in FA pathway function. Consistent with this observation, FA pathway-deficient cells are selectively sensitive to the ATM inhibitor KU-55933. As regards cancer therapy, this is encouraging, as it reveals a potential therapeutic window between normal cells and cancer cells for ATM inhibitors. Moreover, the identification of loss of FANCD2 monoubiquitination or the presence of constitutive ATM activation in tumor tissue may represent useful biomarkers to select patients for ATM inhibitor treatment in future clinical studies.

\section{Methods}

Cell lines and culture conditions. Generation and maintenance of the EUFA326-, EUFA426-, GM6914-, PD20-, DF1179-, EUFA130-, AT22IJET-, HS766T-, and PL-11-derived cell lines has been previously described $(10,30,33,47-49)$. The SV40-transformed DF1179 FANCE mutant fibroblast cell line was functionally complemented with pMMP retroviral vector containing FANCE cDNA, as previously described (49), and grown in Chang medium (Irvine Scientific) with $1 \mu \mathrm{g} / \mathrm{ml}$ puromycin. The HeLa cell line and MEFs established from day $14 \mathrm{Fancg}^{-/}$and $\mathrm{Fancg}^{+/}$embryos were cultured in DMEM medium supplemented with $20 \%$ fetal calf serum.

siRNA library screen. The QIAGEN DNA damage response siRNA library targeting 230 DNA damage response genes was purchased in seven 96-well plates. Each plate also contained 2 GFP-targeted siRNAs, 2 LacZ-targeted siRNAs, and 16 wells containing no siRNA as controls. EUFA326 or EUFA326G cells were seeded in 96-well plates (BD Biosciences) at 1,000 cells per well in $80 \mu \mathrm{l}$ of medium. Twenty-four hours later, a transfection mix of $15.5 \mu \mathrm{l}$ of OptiMEM (Invitrogen), $0.5 \mu \mathrm{l}$ of HiPerFect (QIAGEN), and $4 \mu \mathrm{l}$ of $2 \mu \mathrm{M}$ siRNA oligonucleotide was added to each well. Viability at 96 hours was measured using the CellTiter-Glo Luminescent Cell Viability Assay kit (Promega). The experiment was performed twice to allow statistical analysis of the targets. The corrected viability for each siRNA oligonucleotide was calculated as a percentage of the mean viability of the 16 control wells on each plate. The corrected viability of the EUFA326 cell line was divided by the corrected viability of the EUFA326G cell line to calculate the relative viability for each respective gene target. The mean viability of the EUFA326 relative to the EUFA326G cell line for each gene target, along with the SEM, was calculated from 4 individual corrected viability values that represent duplicate results from the 2 different oligonucleotides on each plate targeting a particular gene.

Crossing of $\mathrm{Fancg}^{+/-} \mathrm{Atm}^{+/-}$mice. Fancg ${ }^{+/-} \mathrm{Atm}^{+/+}$mice, previously generated in our laboratory (50), were crossed with $\mathrm{Fancg}^{+/+} \mathrm{Atm}^{+/-}$mice (51) to generate $\mathrm{Fancg}^{+/-} \mathrm{Atm}^{+/-}$mice. These mice were then interbred and the genotype of the offspring assessed using genomic PCR as previously described $(50,51)$. A statistical comparison of the observed with the predicted genotypes was performed using a 2-sided Fisher's exact test. Significance was set at a $P$ value of less than 0.05 . Animal experiments were approved by the Dana-Farber Cancer Institute Animal Care and Use Committee.

Cell viability assay. Cells were seeded in 96-well plates at a density of 1,000 cells per well on day 1 . To study viability following KU-55933 treatment, cells were treated with KU-55933 (Sigma-Aldrich) at a concentration ranging from $1.5 \mu \mathrm{M}$ to $20 \mu \mathrm{M}$ on day 2. Control wells were treated with DMSO and medium as controls. For viability assays following siRNA treatment, each well was treated with $20 \mathrm{nM}$ siRNA. After 72 hours for KU-55933 treated cells and 96 hours for siRNA treated cells, cellular viability was measured using the CellTiter-Glo Luminescent Cell Viability Assay kit (Promega) following the manufacturer's instructions. The mean cellular viability and SEM were calculated as a percentage of untreated control from 3 separate experiments and plotted using GraphPad Prism version 3 (GraphPad Software).
Comet assay. EUFA326 or EUFA326G cells were seeded at $50 \%$ confluence in 100-mm dishes and transfected with siRNA targeted to ATM or GFP control. After 96 hours, DNA breakage was measured using the neutral Trevigen CometAssay kit as per the manufacturer's instructions. Percentage DNA in comet tails was scored for 100 comets from each cell line using CometScore software version 1.5 (TriTek). The experiment was repeated twice and the data sets combined. The mean percentage DNA per comet tail \pm SEM was calculated using GraphPad Prism software.

Cell cycle analysis, BrdU, and annexin $V$ assays. Flow cytometry and BrdU uptake assays were performed as previously described (8). Cell cycle analysis was performed in 3 separate experiments with cell counts of 20,000 using WinMDI 2.8 software (http://facs.scripps.edu/software.html). Cell death was measured using Annexin V-FITC Apoptosis Detection Kit I (BD Biosciences - Pharmingen) as per the manufacturer's instructions.

Clonogenic assays. Cells were treated for 24 hours with KU-55933 or DMSO control and seeded in $10-\mathrm{cm}$ dishes. Colonies were counted at 14 days as previously described (52). The mean colony count and SEM were plotted using GraphPad Prism software and represent data from 3 separate experiments.

siRNA oligonucleotides. Cell lines were transfected with $20 \mathrm{nM}$ siRNA oligonucleotide using HiPerFect transfection reagent (QIAGEN) following the manufacturer's instructions. The following siRNA target sequences were used: ATM, 5' -AACATACTACTCAAAGACATT-3'; FANCG, 5'-CCCAGGTAATCGAGACACTTA-3'; FANCC, 5' -CGCGAGGACCACCCGATTTAA3'; and GFP, 5'-AAGCAGCACGACTTCTTCAAG-3'.

Generation of the anti-murine Fancd2 polyclonal antibody. A rabbit polyclonal antiserum (G33) against murine Fancd2 was generated using a GSTFancd2 (N-terminal) fusion protein as antigen. Rabbit antiserum was affinity purified using a GST-Fancd2 (N-terminal column).

Immunoblotting. Western blotting was performed as previously described (10). PVDF membranes were probed with the following antibodies: antiATM (1:500; catalog NB100-104; Novus Biologicals), anti-phosphoATM (1:500; catalog ab2888; Novus Biologicals), anti-human FANCD2 (1:1,000; catalog sc-20022; Santa Cruz Biotechnology Inc.), anti-mouse Fancd2 (1:1,000 see Generation of the anti-murine Fancd2 polyclonal antibody), anti-FANCC (1:1,000; ref. 53), anti-FANCG (1:1,000; ref. 54), anti-vinculin (1:2,000; catalog sc25336; Santa Cruz Biotechnology Inc.), anti-phosphoH2AX Ser139 (1:2,000; catalog 07-164; Upstate).

ATM in vitro kinase assay. The ATM kinase assay was performed as previously described (44) with the following modifications. Protein sepharose G beads (GE HealthCare) bound to ATM protein/ATM antibody (ab2617; Abcam) were resuspended in $100 \mu \mathrm{l}$ of kinase buffer and $1 \mu \mathrm{l}$ of recombinant human p53 (BD Biosciences - Pharmingen). Each reaction was incubated for 1 hour at $37^{\circ} \mathrm{C}$ and probed by Western blotting for phospho-p53 Ser15 (1:1,000; catalog 9284; Cell Signaling), p53 (1:1,000; catalog sc-98; Santa Cruz Biotechnology Inc.), and ATM (1:1,000; catalog NB100-104; Novus Biologicals).

Chromosomal breakage analysis. Cells were treated with $20 \mu \mathrm{M}$ KU-55933 for 24 or 48 hours. Chromosomal breakage was analyzed on metaphase spreads as previously described (50).

\section{Acknowledgments}

The authors would like to thank Scott Kern of Johns Hopkins University School of Medicine for supplying the HS766T- and PL-11derived cell lines and Martin Cohn for the EUFA426-derived cell lines. We thank Yosef Shiloh of Tel Aviv University for the AT22IJE-T cell line and Rosalind Segal of Dana-Farber Cancer Institute for the Atmdeficient mice. We also thank Brendan Price, Ronny Drapkin, and Tony Huang of the Dana-Farber Cancer Institute for helpful advice and Patrizia Vinciguerra, Martin Cohn, Kanchan Mirchandani, and Allan Gurtan of the Dana-Farber Cancer Institute for critical reading 
of this manuscript. This work was supported by a Susan G. Komen Breast Cancer Foundation Fellowship (to R.D. Kennedy); NIH grants RO1HL52725, RO1DK43889, PO1150654, P50CA105009-01, PO1HL54785 (to A.D. D’Andrea, E.M. Archila, and M.A. De la Vega), and K23HL068632-03; The V Foundation (to A. Shimamura); Centers for Medical Counter Measures Against Radiation (AI067751 to A.D. D'Andrea, P. Stuckert, and L. Moreau); and The Damon Runyon Cancer Research Foundation Fellowship (to C.C. Chen).
Received for publication December 15, 2006, and accepted in revised form February 13, 2007.

Address correspondence to: Alan D'Andrea, Dana-Farber Cancer Institute, Department of Radiation Oncology, Harvard Medical School, 44 Binney Street, Boston, Massachusetts 02115, USA. Phone: (617) 632-2112; Fax: (617) 632-5757; E-mail: alan_dandrea@ dfci.harvard.edu.
1. Hanahan, D., and Weinberg, R.A. 2000. The hallmarks of cancer. Cell. 100:57-70.

2. Lengauer, C., Kinzler, K.W., and Vogelstein, B. 1998. Genetic instabilities in human cancers. Nature. 396:643-649.

3. Kaelin, W.G., Jr. 1999. Choosing anticancer drug targets in the postgenomic era. J. Clin. Invest. 104:1503-1506.

4. Marnett, L.J., Riggins, J.N., and West, J.D. 2003. Endogenous generation of reactive oxidants and electrophiles and their reactions with DNA and protein. J. Clin. Invest. 111:583-593. doi:10.1172/ JCI200318022.

5. Kennedy, R.D., and D’Andrea, A.D. 2006. DNA repair pathways in clinical practice: lessons from pediatric cancer susceptibility syndromes. J. Clin. Oncol. 24:3799-3808.

6. D'Andrea, A.D., and Grompe, M. 2003. The Fanconi anaemia/BRCA pathway. Nat. Rev. Cancer. 3:23-34.

7. Zou, L., and Elledge, S.J. 2003. Sensing DNA damage through ATRIP recognition of RPA-ssDNA complexes. Science. 300:1542-1548.

8. Andreassen, P.R., D'Andrea, A.D., and Taniguchi, T. 2004. ATR couples FANCD2 monoubiquitination to the DNA-damage response. Genes Dev. 18:1958-1963.

9. Wang, X., Andreassen, P.R., and D'Andrea, A.D. 2004. Functional interaction of monoubiquitinated FANCD2 and BRCA2/FANCD1 in chromatin. Mol. Cell. Biol. 24:5850-5862.

10. Garcia-Higuera, I., et al. 2001. Interaction of the Fanconi anemia proteins and BRCA1 in a common pathway. Mol. Cell. 7:249-262.

11. Taniguchi, T., et al. 2002. S-phase-specific interaction of the Fanconi anemia protein, FANCD2, with BRCA1 and RAD51. Blood. 100:2414-2420.

12. Nakanishi, K., et al. 2002. Interaction of FANCD2 and NBS1 in the DNA damage response. Nat. Cell Biol. 4:913-920.

13. Hussain, S., et al. 2004. Direct interaction of FANCD2 with BRCA2 in DNA damage response pathways. Hum. Mol. Genet. 13:1241-1248.

14. Niedzwiedz, W., et al. 2004. The Fanconi anaemia gene FANCC promotes homologous recombination and error-prone DNA repair. Mol Cell. 15:607-620.

15. King, M.C., Marks, J.H., and Mandell, J.B. 2003. Breast and ovarian cancer risks due to inherited mutations in BRCA1 and BRCA2. Science. 302:643-646.

16. Hahn, S.A., et al. 2003. BRCA2 germline mutations in familial pancreatic carcinoma. J. Natl. Cancer Inst. 95:214-221.

17. Cantor, S., et al. 2004. The BRCA1-associated protein BACH1 is a DNA helicase targeted by clinically relevant inactivating mutations. Proc. Natl. Acad. Sci. U. S. A. 101:2357-2362.

18. Seal, S., et al. 2006. Truncating mutations in the Fanconi anemia J gene BRIP1 are low-penetrance breast cancer susceptibility alleles. Nat. Genet. 38:1239-1241.

19. Couch, F.J., et al. 2005. Germ line Fanconi anemia complementation group $\mathrm{C}$ mutations and pancreatic cancer. Cancer Res. 65:383-386.

20. van der Heijden, M.S., et al. 2004. Functional defects in the Fanconi anemia pathway in pancreatic cancer cells. Am. J. Pathol. 165:651-657.

21. Condie, A., et al. 2002. Analysis of the Fanconi anaemia complementation group A gene in acute myeloid leukaemia. Leuk. Lymphoma. 43:1849-1853.

22. Tischkowitz, M.D., et al. 2004. Deletion and reduced expression of the Fanconi anemia FANCA gene in sporadic acute myeloid leukemia. Lenkemia. 18:420-425.

23. Xie, Y., et al. 2000. Aberrant Fanconi anaemia protein profiles in acute myeloid leukaemia cells. $\mathrm{Br}$. J. Haematol. 111:1057-1064.

24. Pejovic, T., et al. 2006. Cytogenetic instability in ovarian epithelial cells from women at risk of ovarian cancer. Cancer Res. 66:9017-9025.

25. Dhillon, V.S., Shahid, M., and Husain, S.A. 2004. CPG methylation of the FHIT, FANCF, cyclinD2, BRCA2 and RUNX3 genes in Granulosa cell tumors (GCTs) of ovarian origin. Mol. Cancer. 3:33.

26. Narayan, G., et al. 2004. Promoter hypermethylation of FANCF: disruption of Fanconi anemia-BRCA pathway in cervical cancer. Cancer Res. 64:2994-2997.

27. Marsit, C.J., et al. 2004. Inactivation of the Fanconi anemia/BRCA pathway in lung and oral cancers: implications for treatment and survival. Oncogene. 23:1000-1004.

28. Farmer, H., et al. 2005. Targeting the DNA repair defect in BRCA mutant cells as a therapeutic strategy. Nature. 434:917-921.

29. Bryant, H.E., et al. 2005. Specific killing of BRCA2deficient tumours with inhibitors of poly(ADPribose) polymerase. Nature. 434:913-917.

30. Garcia-Higuera, I., Kuang, Y., Denham, J., and D'Andrea, A.D. 2000. The Fanconi anemia proteins FANCA and FANCG stabilize each other and promote the nuclear accumulation of the Fanconi anemia complex. Blood. 96:3224-3230.

31. McCabe, N., et al. 2006. Deficiency in the repair of DNAdamage by homologous recombination and sensitivity to poly(ADP-ribose) polymerase inhibition. Cancer Res. 66:8109-8115.

32. Shiloh, Y. 2003. ATM and related protein kinases: safeguarding genome integrity. Nat. Rev. Cancer. 3:155-168.

33. Ziv, Y., et al. 1997. Recombinant ATM protein complements the cellular A-T phenotype. Oncogene. 15:159-167.

34. Bakkenist, C.J., and Kastan, M.B. 2003. DNA damage activates ATM through intermolecular autophosphorylation and dimer dissociation. Nature. 421:499-506.

35. Singh, N.P., McCoy, M.T., Tice, R.R., and Schneider, E.L. 1988. A simple technique for quantitation of low levels of DNA damage in individual cells. Exp. Cell Res. 175:184-191.

36. Hickson, I., et al. 2004. Identification and characterization of a novel and specific inhibitor of the ataxia-telangiectasia mutated kinase ATM. Cancer Res. 64:9152-9159.

37. Vermes, I., Haanen, C., Steffens-Nakken, H., and Reutelingsperger, C. 1995. A novel assay for apoptosis. Flow cytometric detection of phosphatidylserine expression on early apoptotic cells using fluorescein labelled annexin V. J. Immunol. Methods.
184:39-51.

38. Kaelin, W.G., Jr. 2005. The concept of synthetic lethality in the context of anticancer therapy. Nat. Rev. Cancer. 5:689-698.

39. Lord, C.J., Garrett, M.D., and Ashworth, A. 2006. Targeting the double-strand DNA break repair pathway as a therapeutic strategy. Clin. Cancer Res. 12:4463-4468.

40. Sobeck, A., et al. 2006. Fanconi anemia proteins are required to prevent accumulation of replication-associated DNA double-strand breaks. Mol. Cell. Biol. 26:425-437.

41. Kennedy, R.D., and D'Andrea, A.D. 2005. The Fanconi anemia/BRCA pathway: new faces in the crowd. Genes Dev. 19:2925-2940.

42. Akkari, Y.M., et al. 2001. The $4 \mathrm{~N}$ cell cycle delay in Fanconi anemia reflects growth arrest in late $S$ phase. Mol. Genet. Metab. 74:403-412.

43. Sarkaria, J.N., et al. 1998. Inhibition of phosphoinositide 3 -kinase related kinases by the radiosensitizing agent wortmannin. Cancer Res. 58:4375-4382.

44. Taniguchi, T., et al. 2002. Convergence of the Fanconi anemia and ataxia telangiectasia signaling pathways. Cell. 109:459-472.

45. Riballo, E., et al. 2004. A pathway of double-strand break rejoining dependent upon ATM, Artemis, and proteins locating to gamma-H2AX foci. Mol. Cell. 16:715-724.

46. Morrison, C., et al. 2000. The controlling role of ATM in homologous recombinational repair of DNA damage. EMBO J. 19:463-471.

47. Naf, D., Kupfer, G.M., Suliman, A., Lambert, K., and D'Andrea, A.D. 1998. Functional activity of the Fanconi anemia protein FAA requires FAC binding and nuclear localization. Mol. Cell. Biol. 18:5952-5960.

48. van der Heijden, M.S., et al. 2005. In vivo therapeutic responses contingent on Fanconi anemia/BRCA2 status of the tumor. Clin. Cancer Res. 11:7508-7515.

49. Taniguchi, T., and D'Andrea, A.D. 2002. The Fanconi anemia protein, FANCE, promotes the nuclear accumulation of FANCC. Blood. 100:2457-2462.

50. Yang, Y., et al. 2001. Targeted disruption of the murine Fanconi anemia gene, Fancg/Xrcc9. Blood. 98:3435-3440.

51. Borghesani, P.R, et al. 2000. Abnormal development of Purkinje cells and lymphocytes in Atm mutant mice. Proc. Natl. Acad. Sci. U. S. A. 97:3336-3341.

52. Wilson, J.B., et al. 2001. The Chinese hamster FANCG/XRCC9 mutant NM3 fails to express the monoubiquitinated form of the FANCD2 protein, is hypersensitive to a range of DNA damaging agents and exhibits a normal level of spontaneous sister chromatid exchange. Carcinogenesis. 22:1939-1946.

53. Yamashita, T., Barber, D.L., Zhu, Y., Wu, N., and D'Andrea, A.D. 1994. The Fanconi anemia polypeptide FACC is localized to the cytoplasm. Proc. Natl. Acad. Sci. U. S. A. 91:6712-6716.

54. Garcia-Higuera, I., Kuang, Y., Naf, D., Wasik, J., and D'Andrea, A.D. 1999. Fanconi anemia proteins FANCA, FANCC, and FANCG/XRCC9 interact in a functional nuclear complex. Mol. Cell. Biol. 19:4866-4873. 